\title{
Bacterial Profile and their Antimicrobial Resistance Patterns from Body Fluids at Tikur Anbesa Specialized Hopital, Addis Ababa, Ethiopia
}

Firehiwot Teklehymanot $^{1,2}$, Melese Hailu Legese ${ }^{2^{*}}$ and Kassu Desta ${ }^{2}$

${ }^{1}$ Tikur Anbessa Specialized hospital, College of Health Sciences, Addis Ababa University, Addis Ababa, Ethiopia

${ }^{2}$ Department of Medical Laboratory Science, School of Allied Health Sciences, College of Health Science, Addis Ababa University, Addis Ababa Ethiopia

"Corresponding author: Melese Hailu Legese, Department of Medical Laboratory Science, School of Allied Health Sciences, College of Health Science, Addis Ababa University, Ethiopia, Tel: +251913705279; E-mail: melerose85@gmail.com

Received date: June 20, 2017; Accepted date: August 01, 2017; Published date: August 08, 2017

Copyright: (c) 2017 Teklehymanot F, et al. This is an open-access article distributed under the terms of the Creative Commons Attribution License, which permits unrestricted use, distribution and reproduction in any medium, provided the original author and source are credited.

\section{Abstract}

Background: Infections caused by multidrug resistant (MDR) bacteria remain a public health threat for patients and health care workers. There are scarcity of data on bacterial profiles and their drug susceptibility patterns from body fluids in Ethiopia. Hence, this study aimed at assessing bacterial profiles and their antimicrobial susceptibility patterns (AST) from body fluids at Tikur Anbessa Specialized Hospital (TASH), Addis Ababa, Ethiopia.

Methods: A cross sectional study was conducted from July 2015 to March 2016 by recruiting 384 study participants. Different body fluids were collected and cultured on Blood agar, MacConkey agar and chocolate agar then incubated aerobically and micro-aerobically. Moreover, gram staining, acid-fast staining (AFB) and White blood cell count (WBC) were performed for all collected body fluids sample. Bacterial identification was made using colony morphology, gram stain and biochemical tests. Antimicrobial susceptibility testing was performed on Muller-Hinton agar using disk diffusion method. Data was analyzed using SPSS version 20.

Results: Overall $14.1 \%(n=54 / 384)$ of the body fluids had bacterial growth. Most bacteria were isolated from Cerebrospinal Fluid (CSF) $57.4 \%(n=31 / 54)$ and pleural fluids $33.3 \%(n=18 / 54)$. Of all body fluids, primary gram stain yielded $10.7 \%(n=41 / 384)$ positive results. Majority of body fluids, $44.1 \%(n=173 / 384)$ had abnormal WBC count above 05 cells $/ \mathrm{mm}^{3}$ and $52.6 \%(n=91 / 173)$ of them had polymorphic features. Most frequent bacterial isolates were K. pneumoniae $16.7 \%$ ( $n=9 / 54)$, Coagulase negative Staphylococcus $15.0 \%(n=8 / 54)$ and Pseudomonas spp. $11.1 \%(n=6 / 54)$. Gram-negative and gram-positive bacteria showed highest resistance for Gentamycin $(76 \%)$ and Erythromycin $(59 \%)$ respectively. The MDR level recorded was $75.9 \%(n=41 / 54)$.

Conclusion: Significant numbers of bacteria with high MDR level were isolated from body fluids that call all health care workers and policy makers for concerted efforts for prudent antibiotic use, and limit the transmission of MDR bacteria in hospital and community settings. Regular monitoring of antimicrobial resistance patterns is essential.

Keywords: Bacterial isolates; Multidrug resistance; Pleural fluids; Synovial fluids

\section{Introduction}

Body fluids are important in transporting nutrients as well as waste products, regulating body temperature and assessing respiration process [1]. Generally, body fluids like cerebrospinal fluid (CSF), pleural, peritoneal, synovial and pericardial fluids are naturally free of microorganisms under normal circumstance [2]. However, under infectious condition of central nervous system, peritoneum, joints and other sterile sites, different types of bacteria, fungi, virus and parasites could present and change the physicochemical nature of the body fluids [1-3].

CSF is produced by ultra-filtration or secretion, and circulates through the ventricles and spinal cord and supply nutrients to brain cell and serve as a cushion [3]. Bacterial meningitis is a medical emergency that require urgent rational antibiotics therapy. An estimated 200,000 deaths occur worldwide per year and the mortality rate is higher in developing countries notably in Sub-Saharan Africa, with a range of $16-32 \%$ [4]. On the other hand, peritoneal effusions refers to the detectable and pathologic collection of fluid in the peritoneal cavity and it is the most common complication of cirrhosis and have been a challenge for hospitalized patients [5,6]. Ascites differentiate into transudative as result of systemic conditions such as cirrhosis, heart failure or nephrotic syndrome with low protein concentrations [7] or exudative effusions with high protein concentrations. Similarly, pleural effusions are because of excessive fluid accumulation in the pleural space that has transudative type and exudative type $[7,8]$. Pleural effusions in developed countries are due to malignancy while in developing countries it is mainly due to bacterial infections [9]. Joint fluid is a viscous substance that lubricates joints and many bacteria are responsible for the occurrence of arthritis [6] due to presence of bacterial cell wall fragments and bacterial DNA as indicated from experiments $[10,11]$.

Different pathogenic bacteria like Enterobacteriaceae, Streptococcus pneumoniae, Neisseria meningitides, Group B Streptococci, Listeria monocytogenes, Haemophilus influenzae, Staphylococcus aureus, Acinetobacter and Pseudomonas spp. can invade the various organ and present in the body fluids [5,6,10-12]. Body fluids invaded by such 
bacteria are characterized by having increased WBC count and protein concentration as well as decreased glucose concentration [9]. Bacterial invasion of body fluids cause severe diseases leading to morbidity and mortality unless identified early and treated $[1,2]$. Though isolation and identification of bacterial etiologies are critical for patient mangment [13] developing resistance against commonly used antibiotics are becoming a challenge for treatment success [14]. There are very limited data on the bacterial profiles and their antimicrobial susceptibility patterns from body fluids in Ethiopia in general and in this study site in particular. Hence, assessing bacterial profiles and their AST patterns from body fluids is very crucial to clinicians, microbiologist, and pharmacist and policy makers for proper diagnosis of different infections and for prudent antibiotic use. Therefore, this study aimed to assess the bacterial profiles and their AST patterns from various body fluids submitted to TASH bacteriology laboratory.

\section{Materials and Methods}

A prospective cross sectional study was conducted from July 2015 to March 2016 at TASH. It is the biggest referral hospital of Ethiopia, which is located in the capital city, Addis Ababa. A total of 384 study participants were recruited using convenient sampling technique. The sample size was calculated using a single population proportion formula by taking the value of $\mathrm{P}=0.5$, with marginal error of 0.05 and 95\% Confidence level. All patients, irrespective of age and sex, who gave body fluid samples for microbiological analysis, were included. Patients with history of antibiotics within the last two weeks, contaminated samples, incorrectly labeled and delayed body fluids for more than 2 hrs were excluded. Physicians, following aseptic procedures in sterile tubes, collected body fluids. Demographic information of patients and physical appearance of body fluids (clear, bloody/traumatic, cloudy and clear straw) was recorded using data collection sheet. Immediately after collection, all samples were taken to the bacteriology laboratory for bacteriological culture, primary gram stain, acid-fast stain, total white blood cell (WBC) and differential WBC count.

\section{Microbiological isolation and identification}

Collected body fluids (CSF, pleural, peritoneal and synovial fluids) were inoculated on blood agar (Oxoid, UK), chocolate agar (Oxoid, UK) and MacConkey agar (Oxoid, UK). Blood agar and MacConkey agar were incubated at 35-37C aerobically for 24 hours while chocolate agar were incubated at $35-37^{\circ} \mathrm{C}$ for 72 hours in a candle jar to provide $5-10 \% \mathrm{CO}_{2}$ concentration in order to give chance of growth for microareophilic fastidious bacteria. Plates were examined daily for the growth of bacteria and identifications of bacterial isolates were performed using colony morphology, gram stain from colonies (from culture plates) and conventional biochemical tests. Gram-positive bacteria were identified to species level using catalase, coagulase, latex agglutination test and Pastorex TM staph-plus (Staphylococcus aureus identification) test kits. For identification of gram-negative bacteria, different biochemical tests were used which were indole, triple sugar iron agar, citrate, urea, lysine decarboxylase (LDC) agar and motility. $K$. puemoniae (indole positive) and $K$. oxytoca (indole negative) differentiated based on their indole reaction. Pseudomonas aeroginosa differentiated from other Pseudomonas species using Pseudomonas aeruginosa Screen 80 tablet (Rosco, DK- 2630, and Denmark).

\section{Cell count, gram stain and AFB staining of body fluids}

After inoculation of all body fluids on culture media for bacterial isolation and identification purpose, body fluids samples were diluted and/or directly filled if it is clear and transparent in Improved Neubauer counting chamber and white blood cells were counted in all areas. Then, WBC was calculated per millimeter cube of body fluids as described in literature [15]. Similarly, on the sediment of body fluids three smears were made for differential WBC, gram staining and Acid fast staining methods following the standard procedures. Primary gram stain and acid fast staining were performed directly from collected specimens to compare their diagnostic importance with culture result since they are requested mostly as preliminary diagnostic tools until culture results obtained.

\section{Antimicrobial drug susceptibility testing}

Using Kirby-Bauer disk diffusion method, antimicrobial susceptibility testing performed for each pure isolates that was incubated at $35-37^{\circ} \mathrm{C}$ for $16-18$ hours according to the Clinical and Laboratory Standards Institute (CLSI) [16]. Briefly, 3-5 pure colonies of bacteria were picked from blood agar for Gram positives bacteria, from MacConkey agar for Gram-negative bacteria and chocolate agar for late growers then emulsified in sterile nutrient broth using sterile wire loop. In order to make standardized inoculums size, the bacterial suspension was adjusted to 0.5 McFarland standard using Densitometer and the suspension was swabbed on to Muller-Hinton agar (dispensed on $100 \mathrm{~mm}$ plate). For Streptococcus species, Mueller Hinton agar supplemented with sheep blood were used.

As per the CLSI guideline, bacterial isolates were tested for ampicillin $(10 \mu \mathrm{g}, \mathrm{BD})$, amikacin $(10 \mu \mathrm{g}$, Oxoid $)$, Tobramycin (10 Oxoid), vancomycin (30 $\mu \mathrm{g} \mathrm{BD})$, ceftazidime $(30 \mu \mathrm{g}, \mathrm{BD})$, cefotaxime $(30 \mu \mathrm{g}, \mathrm{BD})$, ceftriaxone $(30 \mu \mathrm{g}, \mathrm{BD})$, chloramphenicol $(30 \mu \mathrm{g}, \mathrm{BD})$, clindamycin $(2 \mu \mathrm{g}, \mathrm{BD})$, gentamicin $(10 \mu \mathrm{g}, \mathrm{BD})$, TMP-SXT $(1.25 \mu \mathrm{g}$ $+23.75 \mu \mathrm{g}, \mathrm{BD})$, tetracycline $(30 \mu \mathrm{g}, \mathrm{BD})$, ciprofloxacin $(5 \mu \mathrm{g}, \mathrm{BD})$, penicillin (10 units, BBL), oxacillin ( $5 \mu \mathrm{g}, \mathrm{BD})$, Rifampin (BD, $5 \mu \mathrm{g})$, Cefuroxime $(30 \mu \mathrm{g}$ BD) and erythromycin (15 $\mu \mathrm{g}, \mathrm{BD})$. Oxacillin (methicillin) susceptibility of Staphylococcus aureus and Coagulase negative Staphylococci was interpreted using $30 \mu \mathrm{g}$ cefoxitin as a surrogate test for Multidrug resistant Staphylococcus species. The zone of inhibition was measured to the nearest millimeter and all bacterial isolates were classified as sensitive, intermediate and resistant.

\section{Quality control and quality assurance}

Standard Operating Procedures (SOPs) were strictly followed verifying that media meet expiration date and quality control parameters. Visual inspections of cracks on media or plastic petridishes, unequal fill, hemolysis, evidence of freezing, bubbles, and contamination was checked. Quality control was performed to check the quality of medium. Each new lot was checked before use by testing Escherichia coli ATCC 25922 and/or Staphylococcus aureus ATCC 25923 standard control strains. We have used known gram positive, gram negative and acid fast positive control slides to check the quality of the various staining methods.

\section{Statistical analysis and interpretation}

The data was entered, cleaned and analyzed using SPSS version 20. The descriptive statistics (mean, percentages or frequency) was calculated. The bi-variant logistic regression analysis was used to see the relation between different study variables. Those variables, which 
Citation: Teklehymanot F, Legese MH, Desta K (2017) Bacterial Profile and their Antimicrobial Resistance Patterns from Body Fluids at Tikur Anbesa Specialized Hopital, Addis Ababa, Ethiopia. Biol Med (Aligarh) 9: 408. doi:10.4172/0974-8369.1000408

Page 3 of 7

showed statistical significant association from bi-variant logistic regression, were further analyzed using multiple logistic regressions. A P-value $<0.05$ was considered as statistically significant.

\section{Ethical clearance}

The Department Research and Ethics Review Committee (DREEC) of Medical Laboratory Science, School of Allied Health Sciences, College of Health Sciences, Addis Ababa University approved the study. Permission letter was also obtained from the study site. All information obtained from patients were kept confidential. Laboratory results were communicated to the requesting physicians on time.

\section{Results}

\section{Socio-demographic characteristics}

A total of 384 patient's body fluids were analyzed for isolation, identification and antimicrobial susceptibility testing. Of these body fluids, cerebrospinal fluids (CSF) was the most frequently encountered body fluids accounting $68.8 \%(n=264 / 384)$ followed by pleural fluid 76 $(\mathrm{n}=19.8 \%)$. The majority of body fluids were clear $61.7 \%(\mathrm{n}=237 / 384)$ in terms of appearance while $19.3 \%(\mathrm{n}=74 / 384)$ body fluids were turbid. Of 384 body fluids, $50.8 \%(n=195 / 384)$ of them were collected from female pateints and 50.8\% $(n=195 / 384)$ were collected from outpatient departments (Figure 1). The age range of study participants was between 24 days-86 years with mean and standard deviation of $15.1 \pm 19.2$ where $70.2 \%(n=271 / 384)$ of them were below 20 years.

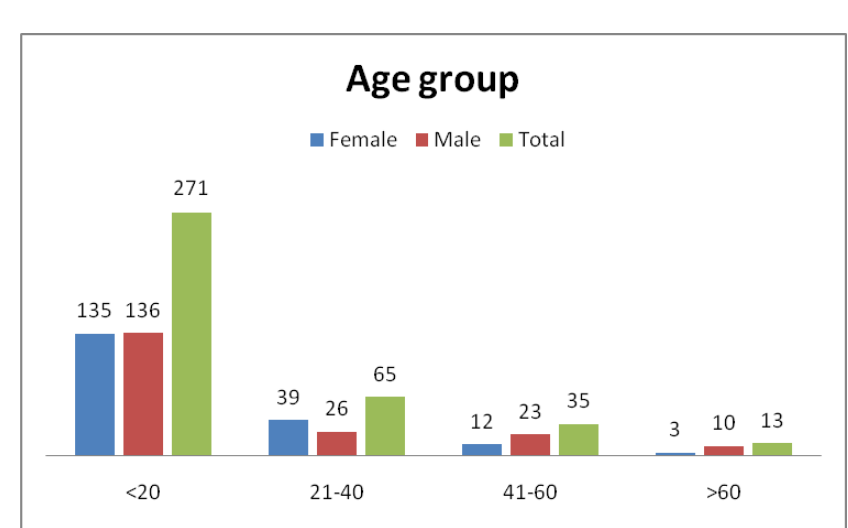

Figure 1: Age in year and sex frequency of pateints analsyed for bacteriology of body fluids at TASH from July 2015 to March, 2016.

\section{Bacterial profiles of body fluids sample}

Overall, $14.1 \%(\mathrm{n}=54 / 384)$ of body fluids had bacterial growth. Of these, $62.2 \%(n=32 / 54)$ of culture positives were from female patients. However, there was no statistical significant difference between gender and culture results $(\mathrm{OR}=0.624,95 \% \mathrm{CI}=0.348-1.119, \mathrm{P}$ value $=0.114)$ (Table 1). Highest bacterial isolation was obtained in age group below 20 years old and statistically significant association was seen between age of patients and culture results $(\mathrm{OR}=1.022,95 \% \mathrm{CI}=1.009-1.036$,
$\mathrm{P}=0.001$. In this study, $51.2 \%(\mathrm{n}=28 / 54)$ culture positive findings were from admitted patients. Gram positive and Gram-negative isolates constitute $40.7 \%(n=22 / 54)$ and $59.3 \%(n=32 / 54)$ respectively with a gram positive to gram-negative ratio of $0.69: 1$. Most bacteria were isolated from CSF samples accounted $57.4 \%(\mathrm{n}=31 / 54)$ followed by pleural fluids $33.3 \%(\mathrm{n}=18 / 54)$ (Table 2). The frequent bacterial isolates were $K$. pneumoniae $16.7 \%(n=9 / 54)$ followed by Coagulase negative Staphylococcus $15.0 \%(\mathrm{n}=8 / 54)$.

\begin{tabular}{|c|c|c|c|c|c|}
\hline \multirow{2}{*}{ Variable } & \multicolumn{2}{|c|}{ Culture results ( $n=38$ ) } & \multirow[t]{2}{*}{$\begin{array}{c}\text { P- } \\
\text { value }\end{array}$} & \multirow{2}{*}{ OR } & \multirow{2}{*}{$95 \% \mathrm{Cl}$} \\
\hline & $\begin{array}{c}\text { Positive no. } \\
\text { (\%) }\end{array}$ & $\begin{array}{c}\text { Negative } \\
\text { no. (\%) }\end{array}$ & & & \\
\hline \multicolumn{6}{|l|}{ Gender } \\
\hline Male & $22(40.7)$ & $173(45.1)$ & 0.114 & 0.624 & $0.348-1.119$ \\
\hline Female & $32(59.3)$ & $157(40.9)$ & & & \\
\hline \multicolumn{6}{|l|}{ Age in years } \\
\hline$<20$ & $27(50.0)$ & $244(63.5)$ & 0.001 & 1.022 & $1.009-1.036$ \\
\hline $21-40$ & 17 (31.5) & $48(12.5)$ & & & \\
\hline $41-60$ & $7(13.0)$ & $48(12.5)$ & & & \\
\hline$>61$ & $3(5.6)$ & $48(12.5)$ & & & \\
\hline \multicolumn{6}{|l|}{ Patient Type } \\
\hline Inpatient & $28(59.9)$ & $155(40.4)$ & 0.05 & 0.651 & $0.424-1.001$ \\
\hline Outnpatient & $26(48.1)$ & $175(45.6)$ & 0.015 & 1.839 & $1.126-3.002$ \\
\hline \multicolumn{6}{|c|}{$\begin{array}{l}\text { Type of body } \\
\text { fluids }\end{array}$} \\
\hline CSF & $31(57.4)$ & $233(60.7)$ & $\begin{array}{l}0.028 \\
9\end{array}$ & 1.208 & $0.852-1.71$ \\
\hline Pleural & 18 (33.3) & $58(15.1)$ & & & \\
\hline Ascetic & $3(5.6)$ & $31(80.7)$ & & & \\
\hline Synovial & $2(3.7)$ & $8(2.1)$ & & & \\
\hline \multicolumn{6}{|c|}{$\begin{array}{l}\text { Appearances of } \\
\text { fluids }\end{array}$} \\
\hline Turbid & $43(79.6)$ & $31(8.1)$ & 0.000 & 1.903 & $1.378-2.628$ \\
\hline Clear & $6(11.1)$ & $231(60.2)$ & & & \\
\hline Clear-straw & $4(7.4)$ & $65(16.9)$ & & & \\
\hline Trauma & $1(1.9)$ & $3(0.8)$ & & & \\
\hline Total & $54(14.0)$ & $330(85.9)$ & & & \\
\hline
\end{tabular}

Table 1: Association of gender, age, patient types, types of body fluids and appearance of body fluids nature with culture results at TASH from July 2015 to March 2016. 
Citation: Teklehymanot F, Legese MH, Desta K (2017) Bacterial Profile and their Antimicrobial Resistance Patterns from Body Fluids at Tikur Anbesa Specialized Hopital, Addis Ababa, Ethiopia. Biol Med (Aligarh) 9: 408. doi:10.4172/0974-8369.1000408

Page 4 of 7

\begin{tabular}{|c|c|c|c|c|c|}
\hline \multirow[b]{2}{*}{ Isolation of bacteria } & \multicolumn{5}{|c|}{ Type of body fluids $n(\%)$} \\
\hline & CSF & $\begin{array}{l}\text { Pleural } \\
\text { fluids }\end{array}$ & $\begin{array}{l}\text { Peritonea } \\
\text { I fluids }\end{array}$ & $\begin{array}{l}\text { Synovia } \\
\text { I fluids }\end{array}$ & Total \\
\hline \multicolumn{6}{|l|}{ Gram negative isolates } \\
\hline K. pneumoniae & $8(1.9)$ & $1(0.2)$ & $0(0)$ & $0(0)$ & $9(2.1)$ \\
\hline Pseudomonas spp. & $2(0.5)$ & $4(.9)$ & $0(0)$ & $0(0)$ & $6(1.4)$ \\
\hline Acinetobacter spp. & $4(0.9)$ & $1(0.2)$ & $0(0)$ & $0(0)$ & $5(1.2)$ \\
\hline E. coli & $0(0)$ & $2(0.5)$ & $1(0.2)$ & $0(0)$ & $3(0.7)$ \\
\hline Citrobacter diversus & $0(0)$ & $0(0)$ & $1(0.2)$ & $0(0)$ & $1(0.2)$ \\
\hline K. rihinoscleris & $0(0)$ & $1(0.2)$ & $0(0)$ & $0(0)$ & $1(0.2)$ \\
\hline K. oxytoca & $1(0.25)$ & $1(0.25)$ & $0(0)$ & $0(0)$ & $2(0.5)$ \\
\hline Enterobacter cloacae & $1(0.25)$ & $1(0.25)$ & $0(0)$ & $0(0)$ & $2(0.5)$ \\
\hline N. meningitides & $1(0.2)$ & $0(0)$ & $0(0)$ & $0(0)$ & $1(0.2)$ \\
\hline$P$. aeruginosa & $0(0)$ & $1(0.2)$ & $0(0)$ & $0(0)$ & $1(0.2)$ \\
\hline P. mirabilis & $0(0)$ & $1(0.2)$ & $0(0)$ & $0(0)$ & $1(0.2)$ \\
\hline Total & $17(53.1)$ & $13(40.6)$ & $2(6.3)$ & $0(00)$ & $32(100)$ \\
\hline \multicolumn{6}{|l|}{ Gram positive isolates } \\
\hline $\begin{array}{l}\text { Coagulase negative } \\
\text { Staphylocoous } \\
\text { (CONS) }\end{array}$ & $8(1.9)$ & $0(0)$ & $0(0)$ & $0(0)$ & $8(1.9)$ \\
\hline S. aureus & $1(0.2)$ & $3(0.9)$ & $0(0)$ & $0(0)$ & $4(0.9)$ \\
\hline S. pneumoniae & $2(0.5)$ & $1(0.2)$ & $0(0)$ & $1(0.2)$ & $4(0.9)$ \\
\hline S.milleri & $0(0)$ & $0(0)$ & $0(0)$ & $2(0.5)$ & $2(0.5)$ \\
\hline Enterococcus spp. & $1(0.25)$ & $1(0.25)$ & $0(0)$ & $0(0)$ & $2(0.5)$ \\
\hline S. viridians & $1(0.2)$ & $0(0)$ & $0(0)$ & $0(0)$ & $1(0.2)$ \\
\hline S. pyogenes & $1(0.1)$ & $0(0)$ & $0(0)$ & $0(0)$ & $1(0.2)$ \\
\hline Total & $14(63.6)$ & $5(22.7)$ & $0(00)$ & $3(13.6)$ & $22(100)$ \\
\hline
\end{tabular}

\section{Gram stain, acid fast stain (AFB), WBC count and appearance of body fluids}

In this study, gram stain were performed directely from all body fluids sample and the yield was $10.7 \%(n=41 / 384)$ of which $56.1 \%$ $(n=23 / 41)$ of the body fluids had gram-negative bacteria. Compared to culture positive results $(\mathrm{n}=54)$, gram stain yielded $75.9 \%(\mathrm{n}=41 / 54)$ positive results while $24.1 \%(\mathrm{n}=13 / 54)$ body fluids had neither gram positive nor gram-negative bacteria. Out of 384 body fluids, $45.1 \%$ $(\mathrm{n}=173 / 384)$ of it had relatively high number of white blood cell count (WBC) above 05 cells $/ \mathrm{mm}^{3}$ and of them $21.1 \%(\mathrm{n}=92 / 384)$ of body fluids had a polymorphic differential WBC features. All body fluids, which were positive by culture, had WBC count of above 20 cells $/ \mathrm{mm}^{3}$ with dominant polymorphic differential features in $72.9 \%(n=39 / 54)$ of body fluids. About $79.6 \%(\mathrm{n}=43 / 54)$ of culture positive body fluids were turbid in appearance and only $11.1 \%$ of body fluids $(n=6 / 54)$ had clear appearance and yield positive cultures. There was significant association between appearance of body fluids and culture results $(\mathrm{OR}=1.64,95 \% \mathrm{CI}=1.903-1.378, \mathrm{P}=0.00$ ) (Table 3 ). Only two body fluids out of 384 were acid-fast positive but none of them was culture positive for any other bacteria.

\section{Antimicrobial drug resistance pattern of bacteria isolated from body fluids}

The antimicrobial drug resistance profiles of bacteria ranged from zero to $100 \%$. The highest resistance rate for gram-negative isolates was recorded for gentamycin (65.6\%), Ampicillin (62.5\%), Ciprofloxacin (53.1\%), Ceftriaxone (50\%) and Tobramycin (50\%) (Table 4). Similarly, gram-positive bacteria showed relatively higher resistance rate to Erythromycin (59\%), Clindamycin (54\%) and Trimethoprim-Sulphamethoxazole (50\%) (Table 5). Of all bacterial isolates, $75.9 \%(n=41 / 54)$ of the bacterial isolates were multidrug resistant (MDR, a bacteria resistant for two or more drugs belonging to different classes of antibiotics). The level of MDR for gram positive and gram-negative isolates was found to be $72 \%(\mathrm{n}=16 / 22)$ and $96 \%$ $(n=29 / 32)$ respectively (Table 6). K. pneumoniae were the dominant isolates and $90 \%(n=8 / 9)$ of these isolates were MDR. From the gram positives isolates, CONS were dominant and all the isolates $(100 \%, 8 / 8)$ were MDR.

Table 2: Frequency of bacteria isolated from different body fluids at TASH from July 2015 to March 2016.

\begin{tabular}{|c|c|c|c|c|c|c|c|c|}
\hline \multirow{3}{*}{$\begin{array}{c}\text { Culture +ve } \\
\text { (54) }\end{array}$} & \multicolumn{2}{|c|}{ Gram stain } & \multicolumn{2}{|r|}{ WBC } & \multicolumn{4}{|c|}{ Body fluid appearance } \\
\hline & Gram bacteria & Gram negative & Polymorphic & Monomorphic & Turbid & Clear & c/straw & Traumatic \\
\hline & 18 & 23 & 39 & 15 & 43 & 6 & 4 & 1 \\
\hline Total & \multicolumn{2}{|c|}{$41^{*}$} & \multicolumn{2}{|r|}{54} & \multicolumn{4}{|c|}{54} \\
\hline
\end{tabular}

Table 3: Frequency of gram Stain, WBC count and sample appearance among the 54 culture positive body fluids at TASH from July 2015 to March 2016. *about 13 body fluids did not have both gram positive and gram negative bacteria.

\begin{tabular}{|c|c|c|c|c|c|c|c|c|c|c|c|}
\hline Bacterial isolate (no.) & AMP & GN & CTX & CRO & CTZ & CRX & TOB & CPR & AMK & CAF & RA \\
\hline K. pneumoniae (9) & $5(55)$ & $9(100)$ & $4(44.4)$ & $6(66.7)$ & $4(44.4)$ & $4(44.4)$ & $4(44.4)$ & $5(55.5)$ & $2(22.2)$ & $4(44.4)$ & NA \\
\hline
\end{tabular}


Citation: Teklehymanot F, Legese MH, Desta K (2017) Bacterial Profile and their Antimicrobial Resistance Patterns from Body Fluids at Tikur Anbesa Specialized Hopital, Addis Ababa, Ethiopia. Biol Med (Aligarh) 9: 408. doi:10.4172/0974-8369.1000408

Page 5 of 7

\begin{tabular}{|c|c|c|c|c|c|c|c|c|c|c|c|}
\hline Acinetobacter (5) & $3(60)$ & $3(60)$ & $2(40)$ & $2(40)$ & $4(80)$ & $2(40)$ & $3(60)$ & $4(80)$ & $4(80)$ & $4(80)$ & NA \\
\hline E. coli (3) & $3(100)$ & $1(33.3)$ & $1(33.3)$ & $1(33.3)$ & $2(66.7)$ & $1(33.3)$ & $2(66.7)$ & $2(66.7)$ & $0(0)$ & $0(0)$ & NA \\
\hline Pseudomonas (3) & $3(100)$ & $3(100)$ & $133.3)$ & $1(33.3)$ & $3(100)$ & $3(100)$ & $1(33.3)$ & $1(33.3)$ & $1(33.3)$ & 1(33.3) & NA \\
\hline P. aeruginosa (1) & $0(0)$ & $0(0)$ & $1(100)$ & $1(100)$ & $0(0)$ & $0(0)$ & $0(0)$ & $1(100)$ & $0(0)$ & $0(0)$ & NA \\
\hline K. oxytoca (2) & $2(100)$ & $1(50)$ & $0(0)$ & $1(50)$ & $0(0)$ & $1(50)$ & $2(100)$ & $0(0)$ & $1(50)$ & $1(50)$ & NA \\
\hline E. cloacae (2) & $1(50)$ & $2(100)$ & $0(0)$ & $1(50)$ & $0(0)$ & $0(0)$ & $2(100)$ & $1(50)$ & $0(0)$ & $0(0)$ & NA \\
\hline C. divorce (1) & $1(100)$ & $1(100)$ & $0(0)$ & $1(100)$ & $0(0)$ & $0(0)$ & $1(100)$ & $0(0)$ & $1(100)$ & $0(0)$ & NA \\
\hline K. rihinoscleris (1) & $0(0)$ & $1(100)$ & $1(100)$ & $1(100)$ & $0(0)$ & $0(0)$ & $1(100)$ & $1(100)$ & $1(100)$ & $1(10)$ & NA \\
\hline P. mirabilis (1) & $1(100)$ & $1(100)$ & $1(100)$ & $1(100)$ & $0(0)$ & $0(0)$ & $1(100)$ & $1(100)$ & $0(0)$ & $0(0)$ & NA \\
\hline N. meningitides (1) & $1(100)$ & NA & $0(0)$ & $0(0)$ & NA & $0(0)$ & NA & $1(100)$ & NA & $0(0)$ & $1(100)$ \\
\hline Total & $20(62.5)$ & $21(65.6)$ & $13(40.6)$ & $16(50)$ & $11(34.4)$ & $11(34.4)$ & $16(50)$ & $17(53.1)$ & $10(31.3)$ & $12(37.5)$ & $1(3.1)$ \\
\hline
\end{tabular}

Note: NA: Not applicable; AMK: Amikacin; AMP: Ampicillin; SXT: Sulfamethoxazole-trimethoprim; CRO: Ceftriaxone; CTX: Cefotaxime; GN: Gentamycin; CTZ: Ceftazidime; CPR: Ciprofloxacin; TOB: Tobramycin; CAF: Chloramphenicol; RA: Rifampicin; R: Resistance.

Table 4: Antimicrobial resistance pattern of gram-negative bacterial isolates $(\mathrm{n}=32)$ from body fluids samples at TASH from July 2015 to March 2016.

\begin{tabular}{|l|l|l|l|l|l|l|l|l|l|l|l|l|}
\hline Bacterial isolates, (no.) & R & AM & P & CTX & CRO & CRX & CLN & ERY & VA & CPR & SXT & OX \\
\hline S. aureus (4) & R & $2(50)$ & $2(50)$ & $2(50)$ & $2(50)$ & $2(50)$ & $2(50)$ & $2(50)$ & NA & $1(25)$ & $3(75)$ & $2(50)$ \\
\hline CONS (8) & R & $5(64)$ & $5(64)$ & $3(38)$ & $3(38)$ & $4(50)$ & $6(75)$ & $6(75)$ & NA & $4(50)$ & $3(38)$ & $3(38)$ \\
\hline S. pneumoniae (4) & R & $1(25)$ & $1(25)$ & $1(75)$ & $1(75)$ & $1(75)$ & $2(50)$ & $2(50)$ & $1(25)$ & $2(50)$ & $2(50)$ & $1(75)$ \\
\hline Enterococcus spp (2) & R & $1(50)$ & NA & NA & NA & NA & NA & NA & $0(0)$ & NA & NA & NA \\
\hline S. viridians (1) & R & $1(50)$ & $0(0)$ & $1(50)$ & $1(50)$ & $1(50)$ & $0(0)$ & $1(50)$ & $0(0)$ & $0(0)$ & $2(100)$ & $1(50)$ \\
\hline S. milleri (2) & R & $1(100)$ & $0(0)$ & $0(0)$ & $0(0)$ & NA & $0(0)$ & $0(0)$ & $0(0)$ & $1(100)$ & $0(0)$ & $0(0)$ \\
\hline S. pyogenes (1) & R & $1(100)$ & $0(0)$ & $0(0)$ & $0(0)$ & NA & $0(0)$ & $0(0)$ & $0(0)$ & $1(100)$ & $1(100)$ & $1(100)$ \\
\hline Total =22 & R & $10(45.4)$ & $8(36.4)$ & $7(31.8)$ & $7(31.8)$ & $8(36.4)$ & $1045.4)$ & $11(50)$ & $2(9)$ & $10(46)$ & $11(50)$ & $9(36)$ \\
\hline $\begin{array}{l}\text { Note: NA: Not applicable; AM: Ampicillin; SXT: Trimethoprim-sulfamethoxazole; CRO: Ceftriaxone; CTX: Cefotaxime; CRX: Cefuroxime; VA: Vancomycin; P: Penicillin; } \\
\text { CPR: Ciprofloxacin; OX: Oxacilin; ERY: Erythromycin; CLN: Clindamycin; R: Resistance. }\end{array}$ \\
\hline
\end{tabular}

Table 5: Antimicrobial resistance pattern of gram-positive bacterial isolates from body fluids samples at TASH from July 2015 to March 2016.

\begin{tabular}{|l|l|l|l|l|l|l|l|}
\hline \multicolumn{7}{|c|}{ Multidrug resistance level of bacterial isolates } \\
\hline Gram Negative & No. tested & R1 & R2 & R3 & R4 & R>4 & Total \\
\hline Pseudomonas spp & $(6)$ & 0 & 2 & 1 & 1 & 1 & 6 \\
\hline P. aeruginosa & $(1)$ & 0 & 0 & 1 & 0 & 0 & 1 \\
\hline E. coli & $(3)$ & 0 & 0 & 0 & 0 & 1 & 5 \\
\hline K. pneumoniae & $(9)$ & 0 & 0 & 0 & 4 & 2 & 9 \\
\hline Acinetobacter & $(5)$ & 0 & 0 & 0 & 2 & & 5 \\
\hline P. mirabilis & $(1)$ & 0 & 0 & 0 & 0 & 1 & 1 \\
\hline K. oxytoca & $(2)$ & 1 & 0 & 0 & 0 & & 1 \\
\hline
\end{tabular}




\begin{tabular}{|c|c|c|c|c|c|c|c|}
\hline Citrobacter diversus & (1) & 0 & 1 & 0 & 0 & 0 & 1 \\
\hline K. rihinoscleris & (1) & 0 & 0 & 0 & 0 & 1 & 1 \\
\hline Enterobacter cloacae & (2) & 0 & 1 & 1 & 0 & 0 & 2 \\
\hline N. meningitides & (1) & 0 & 0 & 1 & 0 & 0 & 1 \\
\hline Total & 32 & $1(3.1)$ & $4(12.5)$ & $4(12.5)$ & $7(21.9)$ & $13(40.6)$ & $29(90.6)$ \\
\hline \multicolumn{8}{|l|}{ Gram positive isolates } \\
\hline S. aureus & (4) & 1 & 2 & 0 & 0 & 0 & 3 \\
\hline S. pneumoniae & (4) & 1 & 0 & 1 & 0 & 0 & 2 \\
\hline Enterococcus spp & (2) & 1 & 0 & 0 & 0 & 0 & 1 \\
\hline CoNS & (8) & 0 & 0 & 2 & 2 & 4 & 8 \\
\hline S. viridians & (2) & 0 & 0 & 0 & 0 & 0 & 0 \\
\hline S. milleri & (1) & 0 & 0 & 1 & 0 & 0 & 1 \\
\hline S. pyogenes & (1) & 0 & 0 & 1 & 0 & 0 & 1 \\
\hline Total & 22 & $3(13.6)$ & $2(9)$ & $5(22.7)$ & $2(9)$ & $4(18.2)$ & $16(72.7)$ \\
\hline
\end{tabular}

Note: R1: resistance to one drug; R2: resistance to two drugs; R3: resistance to three drugs; R4: resistance to four drugs; R>4: resistance to five or more drugs.

Table 6: Multidrug resistance patterns for bacterial isolates from body fluids samples at TASH July 2015 to March 2016.

\section{Discussion}

The overall $14.1 \%(n=54 / 384)$ prevalence of bacteria from body fluids was relatively lower than the finding from Brazil study [17]. This might be due to over diagnosing, prior exposure to antibiotics and emergence of non-infectious conditions like malignancy. Coagulase negative staphylococcus and Acinetobacter spp. were the highest bacterial isolates in our findings that are in agreement with studies conducted in Iran, Korea and Brazil [1,17-19]. The detection of Coagulase negative Staphylococci \& Acinetobacter spp. may be associated with a tendency of these pathogens to cause nosocomial infections, poor infection control practice in hospital, lack of standard facilities, poor sterilization of all gowns and equipment. $S$. pneumoniae and $N$. meningitides were the most common pathogens causing meningitis where our report is in agreement with previous studies from Namibia, Ethiopia, Iran, India and Nepal [9,12,14,20,21]. From CSF samples, Haemophilus influenzae was most frequently isolated bacteria from studies done in Namibia, Iran and Nepal $[9,12,21]$ unlike in our study where no Haemophilus species was detected. This might be due to implementation of Haemophilus vaccine in routine EPI program in Ethiopia since some years back or difference in clinical conditions and the bacteriological culture system. Pseudomomas spp., $S$. aureus and $E$. coli were the commonest bacteria isolated from peritoneal fluids in our study, which is in agreement with studies done in Turkey, Brazil and Nepal $[6,21,22]$. It is known that gram-negative aerobic Enterobacteriaceae from the intestinal lumen can pass to mesenteric lymph nodes or other extra-intestinal sites across the intestinal-mucosal barrier and could appear in body fluids [23]. To the contrary, studies from India and Qatar reported that most frequent isolates of pleural fluids were Streptococcus pneumoniae [22,24].

S. pneumoniae showed $50 \%$ resistance level against penicillin in contrast to other studies done in Namibia, Ethiopia and Iran where
$100 \%$ resistance level to penicillin were reported $[1,12,14]$. However, this big difference can explain in many ways like few strains isolated, change in the resistance profiles of the bacteria and difference in geographical nature of study populations. Similarly, K. pneumoniae showed lower resistance $55 \%$ to ampicillin compared to a study done in Iran that reported $87.5 \%$ resistance to ampicillin [6]. This could be the number of bacterial isolates and difference in the use of antibiotics in each country. The high level of MDR resistance (75\%) in this study is in agreement with a previous study conducted in Gondar, Ethiopia [14]. This high MDR level may be due to inappropriate use of commonly prescribed antibiotics. Previous study in Ethiopia and in Iran reported high level MDR resistance among gram-negative bacteria that is similar to the current study $[1,14]$.

Use of simple gram stain detect the presence of either gram positive or negative bacteria from 41 body fluids (10.7\%) out of 384 total body fluids which is in agreement with a study conducted in India [25]. As compared to culture, gram stain missed only $3.4 \%(n=13 / 384)$ of body fluids, which were positive by culture. This means that, with such limitation, gram stain still provides valuable results as preliminary information until culture results reported. Especially, this is critical in area where culture facility is limited. Interestingly, $72.9 \% \quad(n=39 / 54)$ body fluids, which were culture positive, had high WBC count (above 20 cells $/ \mathrm{mm}^{3}$ ) with dominant polymorphic differential features and majority of them were turbid $(79.6 \%, \mathrm{n}=43 / 54)$. From body fluids which were clear in appearance, $11.1 \%(n=6 / 54)$ of them were culture positive and statistically significant association was observed between body fluids appearance and culture yield (OR=1.64, 95\% $\mathrm{CI}=1.903-1.378, \mathrm{P}=0.00$ ) (Table 3). Only two body fluids out of 384 were acid-fast positive but none of them was culture positive for any bacteria, which might be due to the possibility of disseminated tuberculosis infection. This very low number of AFB results from 384 body fluids might be due to unrelated clinical conditions to 
tuberculosis and underscore the use of AFB stain to rule out tuberculosis infection.

\section{Conclusion}

Significant numbers of both gram negative and gram-positive bacteria were isolated from various body fluids samples. The high level of MDR strains observed urgently call for concerted and immediate attention of health care workers and policy makers for prudent antibiotic use and limits the transmission of MDR bacteria in the hospital and community settings. Moreover, regular monitoring of antimicrobial resistance patterns from all clinical samples including body fluids are essential and the antibiogram should be available to be used by clinicians and policy makers.

\section{Acknowledgment}

We greatly appreciate Addis Ababa University for supporting this study. We are also grateful to the Department of Medical Laboratory Sciences that gave us ethical clearance for this study. Our deep gratitude goes to those study participants and their parents/guardians who showed their willingness to participate in this study by giving written consent.

\section{Conflict of Intereset}

The authors declare that they have no competing interests.

\section{References}

1. Abdinia B, Rezaee MA, Oskouie SA (2014) Etiology and antimicrobial resistance patterns of acute bacterial meningitis in children: a 10-year referral hospital-based study in northwest iran. Iranian Red Crescent Medical Journal 16: e17616.

2. Deb A, Mudshingkar S, Dohe V, Bharadwaj R (2014) Bacteriology of Body Fluids with an Evaluation of Enrichment Technique to Increase Culture Positivity. JEMDS 15230-15238.

3. Hasbun R, Bijlsma M, Brouwer MC, Khoury N, Hadi CM, et al. (2013) Risk score for identifying adults with CSF pleocytosis and negative CSF Gram stain at low risk for an urgent treatable cause. Journal of Infection 67: 102-10.

4. Nwadioha S, Nwokedi E, Onwuezube I, Egesie J, Kashibu E (2013) Bacterial isolates from cerebrospinal fluid of children with suspected acute meningitis in a Nigerian tertiary hospital. The Nigerian postgraduate medical journal 20: 9-13.

5. Syed V, Ansari J, Karki P, Regmi M, Khanal B (2007) Spontaneous bacterial peritonitis (SBP) in cirrhotic ascites: a prospective study in a tertiary care hospital, Nepal. Kathmandu Univ Med J (KUMJ) 5: 48-59.

6. Reginato TJB, Oliveira MJA, Moreira LC, Lamanna A, Acencio MMP, et al. (2011) Characteristics of ascitic fluid from patients with suspected spontaneous bacterial peritonitis in emergency units at a tertiary hospital. Sao Paulo Medical Journal 129: 315-319.

7. Milevoj Kopcinovic L, Culej J (2014) Pleural, peritoneal and pericardial effusions-a biochemical approach. Biochemia Medica 24: 123-137.
8. Tellez-Castillo CJ, Granda DG, Alepuz MB, Lobo VJ, Saiz-Jimenez C, et al. (2010) Isolation of Aurantimonas altamirensis from pleural effusions. J Med Microbiol 59: 1126-1129.

9. Rezaeizadeh G, Pourakbari B, Ashtiani MH, Asgari F, Mahmoudi S, et al. (2012) Antimicrobial Susceptibility of Bacteria Isolated from Cerebrospinal Fluids in an Iranian Referral Pediatric Center. Maedica A Journal of Clinical Medicine 7: 131-137.

10. Moen K, Brun JG, Eribe ER, Olsen I, Jonsson R (2005) Oral bacterial DNAs in synovial fluids of arthritis patients. Microbial ecology in health and disease 17: 2-8.

11. Mardh P, Nilsson F, Bjelle A (1973) Mycoplasmas and bacteria in synovial fluid from patients with arthritis. Ann Rheum Dis 32: 319-325.

12. Mengistu A, Gaeseb J, Uaaka G, Ndjavera C, Kambyambya K, et al. (2013) Antimicrobial sensitivity patterns of cerebrospinal fluid (CSF) isolates in Namibia: implications for empirical antibiotic treatment of meningitis. Journal of Pharmaceutical Policy and Practice 6: 1-10.

13. Riggio O, Angeloni S (2009) Ascitic fluid analysis for diagnosis and monitoring of spontaneous bacterial peritonitis. World J Gastroenterol 15: 3845-3850.

14. Mulu A, Kassu A, Tassema B (2005) Bacterial isolates from cerebrospinal fluids and their antibiotic susceptibility patterns in Gondar University Teaching Hospital, Northwest Ethiopia. Ethiopian Journal of Health Development 19:160-164.

15. Cheesbrough M (2005) District Laboratory Practice in Tropical Countries (2nd edn.). Cambridge University Press, UK.

16. CLSI (2014) Performance Standards for Antimicrobial Disk Susceptibility Tests; Twenty-Third Informational Supplement. CLSI Document 33: M100-S23.

17. Daur AV, Klimak Jr F, Cogo LL, Botão GD, Monteiro CLB, et al. (2006) Enrichment methodology to increase the positivity of cultures from body fluids. Brazilian Journal of Infectious Diseases 10: 372-373.

18. Thomas V (2011) Routine analysis of cirrhotic ascites for evidence of infection-not worth the effort? Indian Journal of Gastroenterology 30: 201-203.

19. Yoon SH, Choi NW, Yun SR (2010) Detecting bacterial growth in continuous ambulatory peritoneal dialysis effluent using two culture methods. Korean J Intern Med 25: 82-85.

20. Thomas K, Kesavan LM, Veeraraghavan B, Jasmine S, Jude J, et al. (2013) Invasive pneumococcal disease associated with high case fatality in India. J Clin Epidemiol 66: 36-43.

21. Shrestha RG, Tandukar S, Ansari S, Subedi A, Shrestha A, et al. (2015) Bacterial meningitis in children under 15 years of age in Nepal. BMC pediatrics 15: 94

22. Dogan M, Kucukkartallar T, Feyzioglu B, Ozdemir M, Baykan M (2013) Isolation of Anaerobic Bacteria from the Peritoneal Fluid of Patient who has Unstrangulated Umbilical Hernia. J Med Microb Diagn 2: 124.

23. de Freitas DG, Gokal R (2005) Sterile peritonitis in the peritoneal dialysis patient. Peritoneal Dialysis International 25: 146-151.

24. Kerr D, Pearson D, Read A (1963) Infection of ascitic fluid in patients with hepatic cirrhosis. Gut 4: 394-398.

25. Vishalakshi B, Hanumanthappa P, Krishna S (2016) A Study on Aerobic Bacteriological Profile of Sterile Body Fluids. Int J Curr Microbiol App Sci 5: 120-126. 